Recepción: 20 / 04 / 2017

Aceptación: 20 / 05 / 2017

Publicación: 15 / 07 / 2017
Ciencias económicas y empresariales Artículo Científico

\title{
Modelo de emprendimiento empresarial para el marketing y comercialización de productos naturales nutricionales en el Ecuador
}

\author{
Entrepreneurship model for the marketing and commercialization of natural \\ nutritional products in Ecuador
}

\section{Modelo de empreendedorismo para o marketing e comercialização de produtos naturais no Equador nutricional}

\author{
Mario F. Miranda-Pazmiño ${ }^{\text {I }}$ \\ miranda_m2008@hotmail.com
}

Mónica M. García-Alvarado II monicamomarina19@yahoo.es

Fátima M. Fernández-Flores III fatimar_1881@hotmail.com

Correspondencia: miranda_m2008@hotmail.com

\footnotetext{
I. Magister en Administración de Empresas Mención en Recursos Humanos y Marketing; Contador Público Autorizado; Contador Público Autorizado, Universidad de Guayaquil, Guayaquil, Ecuador.

II. Magister en Administración de Empresas Mención en Recursos Humanos y Marketing; Ingeniero Comercial, Universidad de Guayaquil, Guayaquil, Ecuador.

III. Magister en Gerencia de Servicios de Salud; Magister en Administración de Empresas con Mención en Recursos Humanos; Diploma Superior en Gestión el Talento Humano; Diploma Superior en Gestión de Desarrollo de los Servicios de Salud; Ingeniera Comercial, Universidad de Guayaquil, Guayaquil, Ecuador.
} 


\section{Resumen}

Es crucial orientar el estudio en procura del reconocimiento de la estructura orgánica de los productos naturales y sus bondades para la salud humana en función de los nutrientes que posee. La aceptación del mercado de productos naturales basados en estándares de calidad generalmente aceptados a nivel internacional, así como también para concienciar y educar al consumidor en el ámbito de la salud orgánica. Es pertinente la presente investigación por estar vinculado a 4 de los 12 objetivos del Plan Nacional de Desarrollo de Ecuador (Plan del Buen Vivir). El análisis de los Resultados demuestran que la combinación del Estudio Técnico con Estudio de Mercado es un aliciente para que la población se concientice de la problemática que enfrenta día a día, donde proliferan productos alterados con químicos perjudiciales para la salud. El Estudio Financiero avala la propuesta al obtener flujos positivos a partir del segundo año, valores crecientes para los siguientes períodos anuales que alientan a que el proyecto se implemente en función de las bondades del producto natural orgánico, obteniendo resultados positivos tanto para el productor, para el consumidor y por consiguiente para la labor de gestión del intermediario (emprendedor-empresario) que permitiría la satisfacción por el valor de uso implícito en el producto dada la rentabilidad social.

Palabras claves: Modelo, Emprendimiento, Productos Naturales, Orgánicos, rentabilidad 


\section{Abstract}

It is crucial to orient the study in search of the recognition of the organic structure of natural products and their benefits to human health based on the nutrients it possesses. The acceptance of the market of natural products based on internationally accepted quality standards, as well as to raise awareness and educate the consumer in the field of organic health. This research is pertinent because it is linked to 4 of the 12 objectives of the Ecuadorian National Development Plan (Plan de Buen Vivir). The analysis of the results show that the combination of the Technical Study with Market Research is an incentive for the population to be aware of the problems that face day to day, where proliferated products proliferate with chemicals harmful to health. The Financial Study endorses the proposal to obtain positive flows from the second year, increasing values for the following annual periods that encourage the project to be implemented based on the benefits of the organic natural product, obtaining positive results for both the producer, The consumer and therefore for the work of management of the intermediary (entrepreneur-entrepreneur) that would allow the satisfaction of the value of use implied in the product given the social profitability.

Key words: Model, Entrepreneurship, Natural Products, Organic, profitability 


\section{Resumo}

É crucial para orientar o estudo busca o reconhecimento da estrutura organizacional de produtos naturais e seus benefícios para a saúde humana em termos de nutrientes que tem. aceitação do mercado de produtos naturais à base de padrões geralmente aceitos de qualidade internacional, bem como para sensibilizar e educar o consumidor em matéria de saúde orgânica. É pertinente a esta investigação ser ligado a 4 dos 12 objetivos do Plano Nacional de Desenvolvimento Equador (Plano de Bem Viver). A análise dos resultados mostra que a combinação de Estudo Estudo de mercado técnico é um incentivo para as pessoas a estar cientes dos problemas que enfrentam todos os dias, onde proliferam alterado com produtos químicos prejudiciais à saúde. O estudo financeira apoia a proposta de alcançar fluxos positivos a partir do segundo ano, valores crescentes para os seguintes períodos anuais incentivar o projeto é implementado com base nos benefícios do produto natural orgânica, resultados positivos tanto para o produtor o consumidor e, portanto, para o trabalho de gestão intermediário (empresário-empregador) permitiria satisfação com o valor de uso implicava o produto dada a rentabilidade social.

Palavras-chave: Modelo, Empreendedorismo, produtos naturais, orgânicos, a rentabilidade 


\section{Introducción}

La promoción y comercialización de los productos alimenticios nutricionales a partir de un Plan de Marketing vinculado a un modelo de Emprendimiento Empresarial tiene amplia potencialidad de cobertura en nuestra sociedad, pero se ve afectada por la proliferación de productos que no tienen claramente identificados los niveles nutricionales, inundando el mercado sin garantizar la eficiencia en la salud de los consumidores.

Por consiguiente, el objetivo de la investigación es Diseñar un Plan Integral de Marketing y Comercialización de Productos Naturales Nutricionales en el Ecuador a partir de un Modelo de Emprendimiento Empresarial específicamente en la zona de influencia de la Aurora y las urbanizaciones satélites a la ciudad de Guayaquil para abrir espacio en el mercado ecuatoriano para educar y concienciar su consumo. Así se determina la aceptación del mercado de productos naturales basados en estándares de calidad generalmente aceptados a nivel internacional.

El Ecuador ha sido un país de producción netamente agrícola desde inicios ancestrales, la colonia y la república, no obstante aquello, en los años de la Revolución Ciudadana, se ha incursionado en la promoción de productos agropecuarios con preservación del medio ambiente y la sustentación como sostenibilidad de las familias campesinas, por lo que se importaron maquinarias agrícolas permitiendo un crecimiento paulatino y fortalecimiento el sector agroindustrial ecuatoriano, dándole un valor agregado a la producción agrícola sin químicos; es así como la Asamblea impulsó leyes que permitieran el crecimiento y consolidación del sector, considerando siempre la preservación del medio ambiente y la tipología de la producción. 


\section{Materiales y métodos}

El enfoque de investigación es Cuantitativo.

Se utilizó la Técnica Documental que permite la recopilación de información para enunciar las teorías que sustentan el estudio de los fenómenos y procesos, se optó por las encuestas que por ser una técnica de adquisición de información de interés sociológico, mediante un cuestionario previamente elaborado, se puede conocer la opinión o valoración del sujeto seleccionado en una muestra sobre un asunto dado (Fichaje y Cuestionario).

Se aplicó la técnica de campo que permitió la observación en contacto directo con el objeto de estudio y el acopio de testimonios que permitan confrontar la teoría con la práctica en la búsqueda de la verdad objetiva.

Se utilizó el Sistema de Información Funcional dirigido a "usuarios finales", Excel de la familia Microsoft para el ingreso, almacenamiento, procesamiento y expulsión de la información.

Por otro lado se utilizó la Metodología Instruccional basada en la Web “Google Docs” para realizar las Encuestas Virtuales que permitieron efectuarlas en diversos puntos de la provincia del Guayas, de conformidad a los contactos registrados por las investigadoras en los dispositivos móviles.

Se hizo el tratamiento de la información desde un enfoque directivo para la toma de decisiones oportuna, reconociéndola como un recurso gerencial al utilizar la plataforma tecnológica tangible e intangible como solución organizacional que permita la consecución del objetivo planteado.

El campo de acción para un plan piloto denominado modelo de Emprendimiento Empresarial se puede ubicar en toda la franja comprendida entre la "Y" del km. 10,5 de las vías a Samborondón, a 
Salitre y a Villa Club, La Joya, El Condado, Villa Italia, Altos del Rio, Volare, siendo el centro de abastecimiento el poblado de la Aurora donde funcionará un local de atención al cliente y almacenamiento transitorio, con RUC de distribuidor artesanal debido a su cercanía a la metrópolis de Guayaquil y en tener una parroquia propia dentro de la aglomeración urbana de Guayaquil (La Aurora),

El cantón Daule, junto con el cantón cercano Nobol, es parte de la Conurbación de GuayaquilDurán-Milagro-Daule la cual les dan una población de 3.021.792. (INEC, 2010)

\section{Resultados}

Los resultados que se obtuvieron del Levantamiento de los Datos fueron diversos, se detallan a continuación.

\section{Sexo:}

261 respuestas de encuestas son mujeres, lo que establece que el 67\% en relación al 33\% (120/361) de varones encuestados.

\section{Ilustración 1: Edad (por rango)}

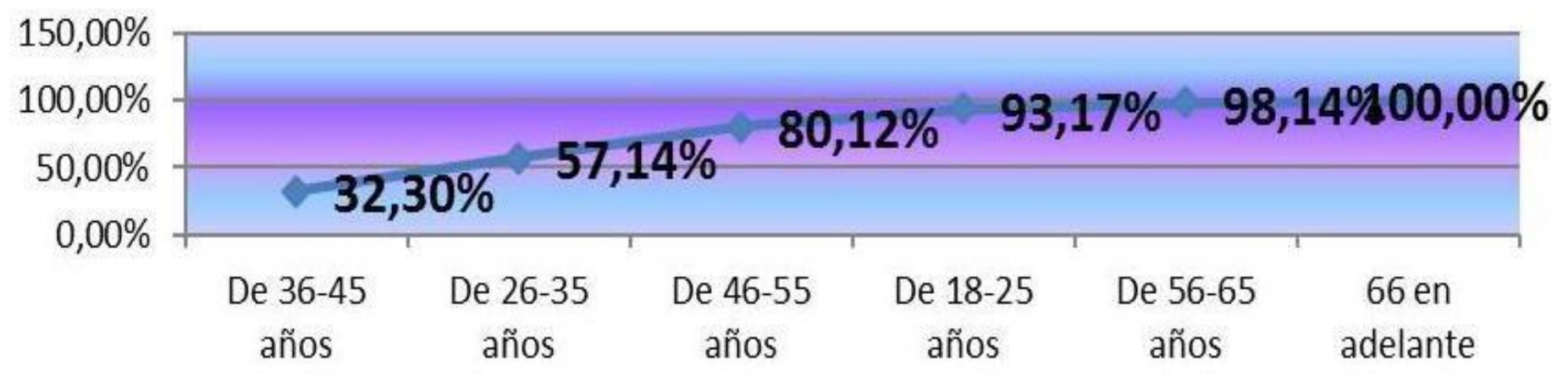

Fuente y Elaboración de Mónica García y Mario Miranda 
La ilustración permite determinar un Pareto casi perfecto en 3 de los 6 rangos de edad en los que se segmentaron a las 361 personas encuestadas que se encuentran distribuidas en los renglones de 3645 años, de 26-35 años y de 46-55 años, en ese orden de mayor a menor cantidad respectivamente, alcanzando un $80 \%$ aproximadamente; mientras que los que pertenecen a los rangos de 18-25 años, de 56-65 y de 66 en adelante, de similar forma secuencial, alcanzan el 20\% restante el total de los encuestados. Estos resultados indican que del total de las personas encuestadas, las que son de edad madura pero jóvenes y las que están por acceder a esa edad madura, son las encargadas de proveerse de alimentos en los diferentes centros de abastos que se analizarán más adelante.

\section{Tabla 1: Estado Civil}

\begin{tabular}{|l|c|c|c|c|c|c|}
\hline Estado civil & Casado(a) & Unión Libre & Soltero(a) & Separado(a) & Divorciado(a) & Viudo(a) \\
\hline Resultado & 192 & 63 & 47 & 31 & 18 & 10 \\
\hline
\end{tabular}

Fuente y Elaboración de Mónica García y Mario Miranda

La mayoría de las personas encuestadas son casadas representan el 53\% (192/361) del total, reconociéndose de esta manera el mercado meta y su segmentación para acceder a la promoción de los productos naturales nutritivos.

\section{Tabla 2: Número de hijos}

\begin{tabular}{|l|l|l|l|l|l|}
\hline Nro de Hijos & $\mathbf{0}$ & $\mathbf{1}$ & $\mathbf{2}$ & $\mathbf{3}$ & $\mathbf{4}$ \\
\hline Respuestas & 32 & 95 & 153 & 54 & 27 \\
\hline
\end{tabular}

Fuente y Elaboración de Mónica García y Mario Miranda 
El $42 \%$ de las personas tienen 2 hijos, lo cual es un excelente indicador para reconocer el tamaño de la familia ecuatoriana asentada en las urbanizaciones satélites a la ciudad de Guayaquil; el siguiente segmento con 1 hijo obtiene el 26\%, ocupando el segundo lugar y con 3 hijos el 15\% en el tercer lugar; sumando estos tres segmentos, se obtiene un Pareto (83\%).

¿Por qué adquiere alimentos procesados, como leche, pollo incubado, galletas, snacks, colas, jugos, etc.? *Obligatorio

Esta es una sociedad de costumbre ya que el 53\% (190/361) adquieren los productos por ese concepto; en segunda instancia se ubica la tendencia de que "el precio es accesible" el 25\%, (92/361) aunque no se tiene la clara la idea de accesibilidad contra qué precio; finalmente el $22 \%$ (79/361) indica que es "bueno para la salud, aunque tampoco hay certeza al respecto, por cuanto no tienen a la mano argumentaciones válidas expresadas en los comentarios.

Estos datos son muy informativos para pretender colocar los productos alimenticios nutritivos en el mercado.

\section{¿Dónde adquiere regularmente los alimentos?}

La tendencia es proveerse de los Supermercados (detallistas, retails) el 49\% del total (177/361), lo que explica la tenencia del consumidor de clase media y media baja, de abastecerse para 7 y hasta 15 días, aunque al tratarse de productos alimenticios perecibles entre 48 y 72 horas, 35\% del total (125/361) accede a los mercados de víveres y de las ferias libres que funcionan cada fin de semana, y solo 59 de los ecuestados van a tiendas, lo que otorga el espacio para colocar los productos naturales paquete de consumo semanal, quincenal, mensualmente, tal cual se podrá corroborar en el análisis de la siguiente figura. 
Tabla 3: ¿Cuántas veces a la semana consume productos perecibles en el corto plazo?

\begin{tabular}{|l|c|c|c|c|}
\hline Frecuencia & $\begin{array}{c}\text { 3 veces a la } \\
\text { semana }\end{array}$ & $\begin{array}{c}\text { 2 veces a la } \\
\text { semana }\end{array}$ & Pasando un día & Todos los días \\
\hline Resultado & 28 & 60 & 81 & 192 \\
\hline
\end{tabular}

Fuente y Elaboración de Mónica García y Mario Miranda

Los resultados indican que no hay una relación directa entre el consumo de productos perecibles en el corto plazo (24 a 72 horas), su consumo debe ser inmediato como lo demuestran los resultados ya que 192 personas indicaron que consumen estos productos todos los días (53\% del total). En su conjunto, estos resultados dan la oportunidad de acceder a un mercado ávido de consumo de productos con altos componentes nutritivos, vegetales y orgánicos.

\section{¿Sabía Ud. que no todos los productos naturales son puros, orgánicos (usan químicos) para anticipar su colocación en los mercados?}

Las personas al desconocer en un 58\% (210/361) de la utilización de los químicos para la rápida maduración y colocación de los productos en los centros de abasto, el mercado meta se torna muy accesible para la colocación de productos naturales nutritivos orgánicos, incluyendo al $42 \%$ (151/361) que aunque conoce de la utilización de químicos, adquieren estos productos más por costumbre, lo cual puede ser revertido si se trabaja en la concienciación de una vida plena en salud.

\section{¿Conoce usted que existen comunidades que se dedican a la siembra de productos orgánicos para preservar la salud en familia?}

Es claro que el desconocimiento de los encuestados abriga esperanzas de que con un buen trabajo de emprendimiento se puede lograr la culturización de una sociedad que está presta a romper paradigmas, ya que el 77\% (278 encuestados)se encuentran en esta posición de desconocimiento de 
producción orgánica y el 33\% (83 encuestados) indicaron que si conocían de productores orgánicos; resultados que potencian el modelo de emprendimiento basado en circuitos cortos como una alternativa de solución a los problemas de salud cardiovasculares, diabetes, desnutrición y stress.

\section{¿Consumiría productos orgánicos?}

El mercado está abierto para los tres segmentos (197/361), el 55\% la mayoría de los encuestados están dispuestos a consumir los productos orgánicos, (85/361) el 23\% aún no está decidido a consumirlo, lo cual es muy interesante por el modelo de emprendimiento a aplicarse en vista de que entre los dos segmentos cubren el 78\% de los encuestados, siendo que una de las fuentes de motivación para la colocación de los productos orgánicos, estriba en la educación para la alimentación saludable en familia.

\section{Motivos por los que consumiría productos orgánicos}

El 51\% (186/361) está convencido de que los productos naturales orgánicos son de mucho beneficio para su salud; el 29\% (104/361) está dispuesto a acceder a una dieta vegetariana, y el 71/361 (20\%) lo haría por padecer enfermedades gastrointestinales. Resultados que demuestran por diferentes causas consumirían un producto natural orgánico es más beneficioso para la salud de las personas que el producto tradicional, precisamente por hacer frente a las principales enfermedades que proliferan en la sociedad ecuatoriana, por la mala, pésima alimentación producto de incultura.

\section{Plan de Marketing basado en Productos Naturales Nutricionales de Circuitos Cortos de Producción Agrícola Familiar.}

En la producción agrícola, los circuitos cortos son y representan potenciales oportunidades para diversificar la producción, capturar un mayor valor y asegurar un ingreso más estable. Desde el 
punto de vista de las comunidades locales, son vistos como una forma de relocalización de las cadenas de valor que trata de mantener el valor en los territorios, generando empleos, capturando valor a partir de activos inmateriales (marca, anclaje territorial), valorizando el patrimonio y convirtiéndose en una capacidad de producción dinámica, manteniendo a la población en la zona de producción (en su propio territorio).

Los circuitos de proximidad o circuitos cortos son una forma de comercio basada en la venta directa de productos agrícolas frescos o de temporada. Prácticamente los productores y consumidores se encuentran muy cercanos geográficamente, en las relaciones comerciales entre ambas partes, aparece en el peor de los casos un intermediario.

\section{Figura 1: Factores del modelo de emprendimiento empresarial}

FACTORES DE

PROXIMIDAD:

Geográfica

Organizacional
Producción de

Circuitos Cortos

sin Químicos
Comercialización

Interna y Externa

de Circuitos

Cortos

\section{Mercado Meta}

El mercado meta son la urbanizaciones ubicadas en la franja norte aledaña a la ciudad de Guayaquil, las llamadas urbanizaciones privadas satélites que se encuentran ubicadas a partir de la "Y" del kilómetro 10.5 de la vía a Samborondón, Salitre y la Aurora.

\section{Estrategias de Penetración en el mercado local}

- Lanzar los productos naturales en el mercado meta, informando sobre su valor nutricional y calidad. 
- Utilizar empaques llamativos, innovadores que atraigan al consumidor.

- $\quad$ Inclusión de servicios adicionales como entrega a domicilio.

- Lanzar el producto a un precio que no supere el 5\% del total del costo total de producto en el primer año.

- Colocar promociones como cupones de descuento para las futuras compras, dos por uno, rebajas del $20 \%$ de descuento, combos, entre otras.

- Oferta del producto vía internet como sitio web, llamadas telefónicas, envío de correos electrónicos masivos, visitas a domicilio y redes sociales.

- Colocación del producto abriendo mercado con degustación en todas las urbanizaciones del circuito descrito (acceso a la base de a través de la administración, efectuando alianza estratégica con cada directiva).

- Colocación de puestos de degustación en los centros de distribución de los productos tales como hoteles, restaurantes y tiendas especializadas.

- Apertura de un local como centro de acopio en la Aurora, parroquia ubicada en el centro neurálgico de comercialización de alimentos.

\section{Relaciones Comerciales}

La modalidad de compra ha de ser directa, de los emprendedores comerciantes a los productores, bajo el modelo de Emprendimiento Empresarial para la Comercialización de Productos Naturales, lo cual determina un rol importante en la comercialización de productos en el mercado interno.

Se potenciaría un canal de comercialización muy útil para los pequeños productores (Alianza Estratégica para la adquisición de su producción), en cualquiera de sus tipos, considerando que los pagos son en efectivo. 


\section{Herramientas de Colaboración para la Comercialización}

Se deberá formalizar grupos de colaboración (Directivas de las Asociaciones de Propietarios de las Urbanizaciones del Sector), que viabilicen la venta en pequeña escala, para lo cual se concienciará la “alimentación como medicina integral".

Este modelo permitirá cambiarlos hábitos alimenticios de una comunidad incipiente, joven, trabajadora, educada, a la cual no es difícil formalizarla en hábitos alimenticios saludables.

El beneficio de los productores agrícolas será acorde a su aportación inicial en la cadena de valor, redireccionando rutas de distribución hacia una distribución consistente que no mira solamente la rentabilidad sin antes analizar la utilidad para la sociedad.

Asimismo, se valoriza tanto la proximidad geográfica, buscando que las distancias entre producción y consumo sean reducidas, como la proximidad de identidad cultural, es decir, los productos del territorio

\section{Proyección de ingresos}

\begin{tabular}{|l|l|l|l|l|l|}
\hline Año base 2016 (en \$) & Año 1 & Año 2 & Año 3 & Año 4 & Año 5 \\
\hline Ingresos Mensuales & $2.150,00$ & $3.270,00$ & $4.530,00$ & $5.390,00$ & $6.850,00$ \\
\hline INGRESOS ANUALES & $25.800,00$ & $39.240,00$ & $54.360,00$ & $64.680,00$ & $82.200,00$ \\
\hline
\end{tabular}

Fuente y Elaboración de Mónica García y Mario Miranda

En el ámbito financiero, los resultados son alentadores a pesar de trabajar en un escenario pesimista, contando con un Capital Propio de US\$ 5.000 y un apalancamiento de US\$27.300,00 para la compra de una camioneta para el transporte y logística en el traslado, marketing, venta y colocación 
de los productos naturales orgánicos, así como en las diferentes charlas que se programarán para el efecto.

Solamente el primer año arroja déficit, lo que es normal en este tipo de inversiones, pero a partir del segundo año se obtienen flujos positivos en donde comienza el proceso de fidelización de los clientes-consumidores y a partir del tercer año los productos naturales orgánicos se consolidan en este incipiente mercado.

Los Indicadores de riesgo muestran un escenario muy sensible a cambios en los ingresos, en función de cambios en los precios como también por cambios en las cantidades vendidas.

La tendencia indica que la empresa es rentable, dada una utilización eficiente de sus activos en función de sus utilidades. Su evolución es latente y con tendencia al alza, ya que el rendimiento sobre el capital invertido en cada nuevo período es cada vez más alto.

Los resultados demuestran la aceptación de la Hipótesis planteada "Si se analiza el mercado de productos nutricionales, se puede contribuir con un Plan de Marketing para socializarlo a los microempresarios”, en función de que en el Análisis de los Resultados se demostró que el mercado está abierto para los tres segmentos (197/361), el 55\% la mayoría de los encuestados están dispuestos a consumir los productos orgánicos, y (85/361) el 23\% aún no está decidido a consumirlo, lo cual eleva la tendencia a $88 \%$, que garantiza la existencia de un mercado potencial para la comercialicen de productos naturales nutritivos. 


\section{Conclusiones.}

El modelo de Emprendimiento Empresarial se fundamenta en la producción y comercialización de Circuitos Cortos en donde no existen intermediarios y en el mejor de los casos un solo intermediario, que es el lugar que se pretende ubicar a los emprendedores.

La participación del intermediario (emprendedor empresario) se circunscribe en una logística combinada de transporte, acercamiento al territorio de producción, estructuración de la Red de Consumidores, utilización de los Sistemas de Información para la Gestión del Conocimiento del mercado meta, propiciando el objetivo de satisfacción de las partes, es decir, tanto de las Familias Productoras como de la Red de Consumidores y obteniendo estos intermediarios (EmprendedoresEmpresarios) una cuota de rentabilidad socialmente responsable que le permita cubrir sus costos operativos y gastos administrativos, agregando valor tanto para la comunidad productora, los clientes-consumidores, cuanto para sí mismo.

Es importante reconocer que una de las limitaciones visibles es la integración de las pequeñas producciones familiares que permitan abastecer frecuentemente y de manera consistente y sostenible a la Red de Consumidores (mercado meta) ubicada en la zona de urbanizaciones de clase media cercanas a los territorios de producción (Salitre, Samborondón, Daule).

Por el lado del mercado meta, los residentes de las urbanizaciones son generalmente trabajadores con relación de dependencia en su mayoría, y que tienen educación de tercer nivel con una cultura pujante que se sostiene a través de la aportación de prácticamente todos los miembros adultas de cada familia. 
El modelo también considera la capacitación y educación de esta red para que asimilen la propuesta de alimentarse en salud, tarea nada sencilla considerando el arraigo de costumbres y temor a lo desconocido.

El gobierno debe continuar impulsando los programas de "alimentación en salud" para que se sustente tanto la economía agrícola como la salud de los clientes-consumidores y población en general.

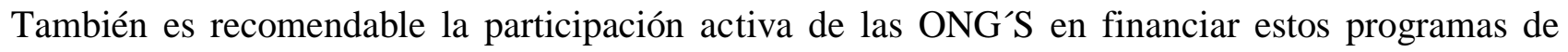
alimentación natural nutritiva.

Aprovechar la Biodiversidad existente en todo el territorio ecuatoriano, la producción agrícola natural orgánica puede segmentarse en función de las cuatro regiones en que está distribuido el territorio ecuatoriano.

Finalmente la empresa privada debe fomentar créditos vinculados al sector agrícola, otorgando preferencias a la producción orgánica de productos nutritivos pue permitan una producción sustentable y sostenible.

\section{Bibliografía.}

Bustos, S. (2014), "Escuelas y circuitos cortos: el programa „Eat Healthy, Live Healthy" en los Estados Unidos”, Memoria del seminario sobre circuitos cortos, septiembre de 2013. Santiago, Chile.

Cabrero, J. (2006). Marketing de clientes. Madrid: McGraw-Hill.

Corporación Financiera Internacional. (2007). Relaciones con la comunidad y otros actores sociales: Manual de prácticas recomendadas para las empresas que hacen negocios en mercados emergentes. Washington.

FAO (2014), Agricultura Familiar en América Latina y el Caribe: Recomendaciones de Política. Salcedo, S. y Guzmán, L.,eds. Oficina Regional de la FAO para América Latina y el Caribe. Santiago, Chile.

Faus, M. (2004). Temas claves en el marketing relacional. Madrid, España: McGraw-Hill. 
Modelo de emprendimiento empresarial para el marketing y comercialización de productos naturales nutricionales en el Ecuador

González, M. d. (18, 19 y 20 de Octubre de 2005). Jornadas de economia alternativa y solidaria. Recuperado el 09 de 06 de 2015, de http://www.hegoa.ehu.es/congreso/bilbo/doku/bat/responsabilidadsocialc orporativa.pdf

Hernández. (2014). Metodología de la Investigación. En H. S. Roberto, Metodología de la Investigación.

Hill, M. (25 de 09 de 2011). Libroos.es. Recuperado el 09 de 06 de 2015, de http://www.mcgrawhill.es/bcv/guide/capitulo/8448176081.pdf

Jiménez, A. (2000). Gestión del marketing social.España: McGraw-Hill.

Kebir L., Torre A. (2013), Geographical proximity and new short supply food chains, in Lazzeretti L. (ed), Creative Industries and Innovation in Europe, Concepts, Measures, and Comparative Case Studies, Routledge, N. York, 328 p.

Midoux, B. (2014), "Politicas para promover los circuitos cortos: la experiencia del Ministerio de Agricultura de Francia"

Observatorio europeo LEADER (2000), Comercialización de los productos locales: Circuitos cortos y circuitos largos, cuaderno de la Innovación n 7 , Innovación en el medio rural, Francia.

Responsabilidad Social (2013). Obtenido de http://www.revistalideres.ec/lideres/empresas-integranresponsabilidad-social-corporativa.html

Romero, G. (2014), “Agricultura familiar, circuitos cortos y el Programa Nacional de Alimentación Escolar Qali Warma, Perú”, Septiembre de 2013. Santiago, Chile.

Siliprandi, E. (2014), "Los programas de compras públicas de Brasil: escuelas y circuitos cortos”, Septiembre de 2013. Santiago, Chile.

Verón. (10 de 05 de 2015). Guia Empresa XXI. Obtenido de Guia Empresa XXI: http://www.guiaempresaxxi.com/opinion/empresas-socialmente-responsables.html

Vives, A., \& Peinado-Vara, E. (2011). La responsabilidad social de la empresa en América Latina.Washington, D.C: Banco Interamericano de Desarrollo. 